\title{
MiR-26a Mediates Ultraviolet B-Induced Apoptosis by Targeting Histone Methyltransferase EZH2 Depending on Myc Expression
}

\author{
Ting Zhang Hua Qian Cui Hu Hui Lu Ji-Bing Li Ya-Fen Wu Wei Li \\ Department of Dermatology, Children's Hospital of Soochow University, Suzhou, Jiangsu, China
}

\author{
Key Words \\ Skin cancer $・ \mathrm{MiR}-26 \mathrm{a} \cdot \mathrm{EZH} 2 \cdot \mathrm{MYC} \cdot \mathrm{UVB} \cdot$ Apoptosis
}

\begin{abstract}
Background/Aims: Ultraviolet B (UVB) damage is the most essential etiological factor in skin carcinogenesis, and apoptosis leads to the efficient elimination of UVB-damaged cells. However, the mechanisms underlying resistance to UVB-induced apoptosis remain unclear. Methods: $\mathrm{HaCaT}$ and A431 cells were used in the present study. Quantitative real-time PCR, single cell PCR, and western blotting were used to examine cancer-related gene expression at the mRNA and protein levels. Results: We report that miR-26a, which is upregulated upon UVB irradiation, promotes UVB-induced apoptosis in $\mathrm{HaCaT}$ cells by targeting the histone methyltransferase EZH2. Moreover, the UVB/miR-26a/EZH2 regulatory axis largely depends on the MYC expression level. Interestingly, treatment with EZH2 inhibitors significantly enhanced UVB-induced apoptosis. Conclusion: miR-26a/EZH2 might be potential targets for skin cancer prevention and therapy.
\end{abstract}

\section{Introduction}

Ultraviolet (UV) radiation causes DNA damage and apoptosis, and can lead to skin carcinogenesis, aging, hyperplasia, and immune tolerance [1,2]. Skin cancer is the most commonly occurring cancer, with increasing morbidity in light-skinned individuals [3], and ultraviolet B (UVB, 280-320 nm) radiation is a major environmental factor for human non-melanoma skin carcinogenesis [4]. Although the pathophysiology of skin cancer is multifactoral for the difference of moisture content, smoothness, elasticity, and thickness of epidermis [5], the mechanism of skin carcinogenesis has been fully investigated.

DNA is the main target of UVB, which causes somatic mutations and aberrant expression of tumor suppressors and oncogenes [6]. For instance, UVB-induced p53 mutations have been found in over $90 \%$ of human skin cancers, and abnormal p53 activity is a major factor 


\section{Cellular Physiology Cell Physiol Biochem 2017;43:1188-1197

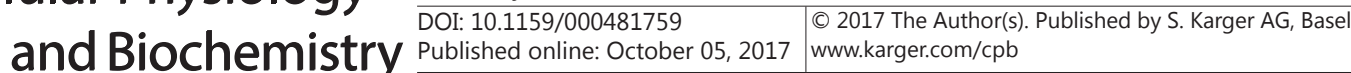 \\ Zhang et al.: Mir-26a Mediates Apoptosis in UVB Irradiated Cells}

in skin cancer onset and progression [7]. Apoptosis, or programmed cell death, is the main cellular response to UV radiation. It has been proposed that failure to repair damaged DNA or eliminate damaged cells by apoptosis, which leads to the replication of damaged DNA and proliferation of damaged cells, is the main cause of skin carcinogenesis [8]. The tumor suppressor $\mathrm{p} 53$ acts as a proofreading factor to eliminate rather than repair severely damaged cells through apoptotic pathways [7]. Cells that escape this proofreading generate skin cancer cells, but the underlying mechanism remains unclear.

MicroRNAs (miRNAs) are endogenous noncoding RNAs that regulate many cellular processes by targeting specific proteins for degradation. They are pivotal for sensing environmental stresses, such as UV radiation [9-11]. Previously, we identified dozens of miRNAs that are transcriptionally altered in response to UVA irradiation [12], and found that miR-23a, miR-1246, and miR-141 have roles in UV-induced apoptosis and aging [13-15]. miR-26a is a well-established tumor suppressor that targets multiple oncoproteins, such as CDC6, HMGA1, FGF9, and AKT [16-19]. However, it remains unknown whether miR-26a is involved in UVB-induced apoptosis and skin tumorigenesis.

Here, we assessed the effect of UVB irradiation on miR-26a expression in HaCaT and A431 cells, and found that miR-26a was strongly induced by UVB stimulation, in parallel with apoptosis. EZH2 was identified as a MYC-dependent miR-26a target involved in UVBinduced apoptosis. EZH2 inhibition enhanced UVB-stimulated apoptosis, suggesting that EZH2 may be a promising target for the prevention and treatment of skin cancer.

\section{Materials and Methods}

Cell culture and UVB irradiation

HaCaT and A431 keratinocyte cell lines were purchased from American Type Culture Collection (ATCC) and cultured in standard medium conditions as recommended. UVB irradiation was conducted as previously described [14].

\section{RNA isolation and quantitative real-time PCR}

The TRI Reagent Kit (Ambion, USA) was used to isolate total RNA, according to the manufacturer's instructions. Expression of miR-26a was analyzed using the TaqMan MicroRNA Reverse Transcription Kit and SYBR Green Real-time PCR Master Mix (Toyobo, Japan). The relative expression level of miR-26a was normalized to that of an endogenous control (U6). For detection of gene expression, reverse transcription was performed using SuperScript III (Invitrogen, USA), and SYBR Green real-time PCR master mix (Toyobo, Japan) was used for real-time PCR analysis. Relative gene expression levels were normalized to GAPDH. The primer sequences used were as follows:

miR-26a forward: $5^{\prime}$-CTGTCAACGATACGCTAC- $3^{\prime}$

miR-26a reverse: 5'-GTAATCCAGGATAGGCTG-3'

EZH2 forward: $5^{\prime}$ - TACTTGTGGAGCCGCTGAC-3'

EZH2 reverse: 5' - CTGCCACGTCAGATGGTG-3'

MYC forward: 5'-AATGAAAAGGCCCCCAAGGTAGTTATCC-3'

MYC reverse: 5'-GTCGTTTCCGCAACAAGTCCTCTTC-3'

CDK4 forward: 5'-GGGGACCTAGAGCAACTTACT-3'

CDK4 reverse: 5'-CAGCGCAGTCCTTCCAAAT-3'

CDK6 forward: 5'-GCTGACCAGCAGTACGAATG-3'

CDK6 reverse: 5'-GCACACATCAAACAACCTGACC-3'

p21 forward: 5' - GAGGCCGGGATGAGTTGGGAGGAG-3'

p21 reverse: $5^{\prime}$ - CAGCCGGCGTTTGGAGTGGTAGAA- $3^{\prime}$

BAX forward: 5'-CCCGAGAGGTCTTTTTCCGAG-3'

BAX reverse: 5'-CCAGCCCATGATGGTTCTGAT-3'

BCL2 forward: 5'-GGTGGGGTCATGTGTGTGG-3'

BCL2 reverse: $5^{\prime}$-CGGTTCAGGTACTCAGTCATCC-3'

U6 forward: 5'-TGCGGGTGCTCGCTTCGGCAGC-3' 


\title{
Cellular Physiology Cell Physiol Biochem 2017;43:1188-1197

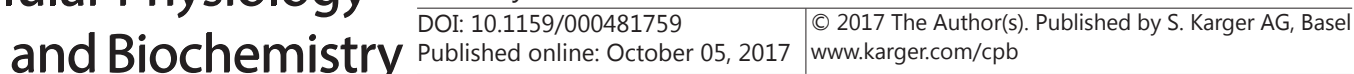

\author{
U6 reverse: $5^{\prime}$-CCAGTGCAGGGTCCGAGGT-3' \\ GAPDH forward: 5'-GATTCCACCCATGGCAAAT TC- $3^{\prime}$ \\ GAPDH reverse: 5'-AGCATCGCCCCACTTGATT-3'
}

\section{Apoptosis assays}

The proportion of apoptotic cells upon UVB treatment was detected at indicated time points using an EPICS Elite ESP Flow Cytometer (Beckman-Coulter, USA), as previously reported [14].

\section{Western blot analysis}

Protein samples were prepared and subjected to sodium dodecyl sulfate-polyacrylamide gel electrophoresis (SDS-PAGE) separation as previously reported [15]. Anti-EZH2 (1:1000, BD Bioscience) and anti-GAPDH (1:4000, Santa Cruz Biotechnology, USA) primary antibodies were used with horseradish peroxidase (HRP)-conjugated secondary antibody (1:7000, Sigma). The blots were developed using an enhanced chemiluminescence kit (Pierce, USA).

\section{Luciferase assay}

The $3^{\prime}$-untranslated region (3'-UTR) of human EZH2 was amplified from genomic DNA and cloned into an miRNA luciferase reporter vector, as previously reported [15]. The luciferase reporter construct pRL-TK was co-transfected with RNA oligonucleotides or expression vectors into HaCaT cells for $48 \mathrm{~h}$, after which luciferase reporter activity was determined using a dual luciferase reporter assay system (Promega, USA).

\section{Cell transfection}

Cells were transfected with expression plasmids or RNA oligonucleotides using Lipofectamine 3000 (Invitrogen, USA) following the manufacturer's protocol. Briefly, plasmids were mixed with equal amounts of P3000 and Lipofectamine 3000 in Opti-MEM (Gibco) and incubated in culture medium at room temperature for $10 \mathrm{~min}$. Cells were harvested for analysis $48 \mathrm{~h}$ after transfection.

\section{Single cell PCR}

HaCaT cells treated with UVB were trypsinized to isolate single cells, which were randomly selected for single cell reverse transcription using the Smart-seq2 method, as described previously [20].

\section{Data analysis}

All presented results (mean \pm SD) were repeated independently at least three times, and data were analyzed statistically with the Student's $t$-test. $p<0.05$ was considered statistically significant.

\section{Results}

UVB-induced apoptosis is promoted by miR-26a in HaCaT cells

Several reports have demonstrated roles for miR-26a in various cancers [16-19], but the relationship between miR-26a and UVB-induced apoptosis remains unclear. To test for changes in miR-26a expression in response to UVB-induced apoptosis, HaCaT cells were exposed to UVB irradiation in a time-dependent manner. As expected, apoptosis increased in HaCaT cells upon UVB irradiation, with approximately 26\% apoptotic cells after $48 \mathrm{~h}$ exposure (Fig. 1A). The expression of miR-26a, relative to U6, gradually increased after UVB irradiation (Fig. 1B), along with increased expression of the pro-apoptotic gene BAK (Fig. 1C). This indicates that miR-26a might be positively correlated with UVB-induced apoptosis.

To test this hypothesis, HaCaT cells were transfected with a miR-26a mimic, resulting in significant overexpression of miR-26a (Fig. 1D). Control or miR-26a-overexpressed cells were subjected to UVB irradiation for $24 \mathrm{~h}$ and apoptosis was examined. Notably, miR-26a overexpression strongly promoted apoptosis in the presence or absence of UVB irradiation (Fig. 1E). Consistently, miR-26a overexpression increased the expression of the pro-apoptotic proteins BAK and BAX, while expression of the anti-apoptotic protein BCL2 was decreased (Fig. 1F). To determine whether miR-26a was necessary for UVB-stimulated apoptosis, an 


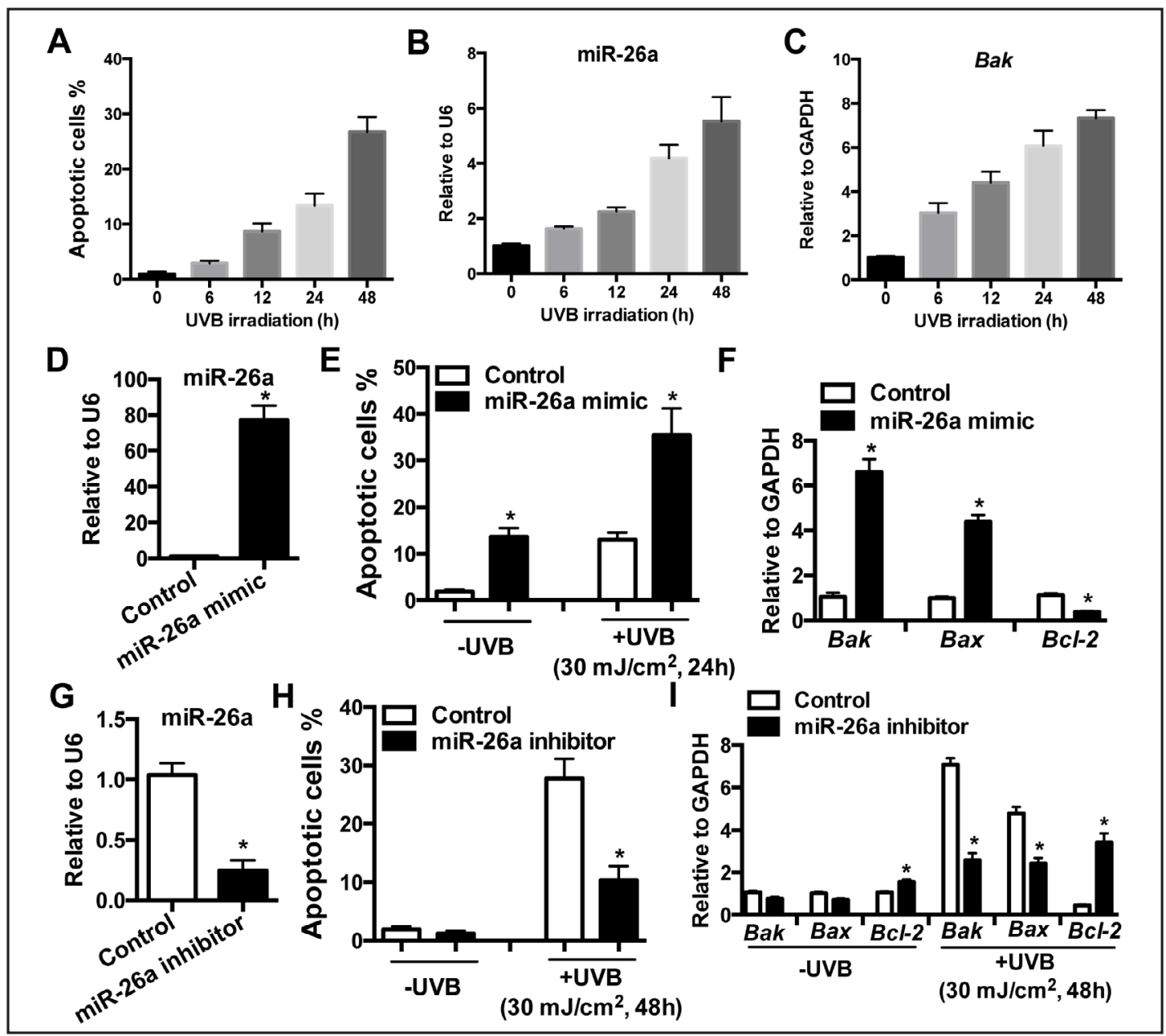

Fig. 1. UVB-induced apoptosis is promoted by miR-26a (A) Apoptotic analysis of HaCaT cells irradiated with $30 \mathrm{~mJ} / \mathrm{cm}^{2}$ UVB for $0,6,12,24$, and $48 \mathrm{~h}$. (B-C) UVB-treated cells were collected for reverse transcription and real-time PCR analysis of miR-26a (B) and BAK (C) expression at the indicated time points. (D) The overexpression of miR-26a was examined by real-time PCR in control or miR-26a mimic-transfected HaCaT cells. (E) Control or miR-26a mimic-transfected HaCaT cells in the absence or presence of $30 \mathrm{~mJ} / \mathrm{cm}^{2} \mathrm{UVB}$ for $24 \mathrm{~h}$ were subjected to apoptotic analysis. (F) Real-time PCR analysis of BAK, BAX, and BCL2 in control or miR-26a mimic-transfected HaCaT cells irradiated with UVB for 48 h. (G) Knockdown efficiency, determined by real-time PCR, in control and miR-26a inhibitor-transfected HaCaT cells. (H) Apoptotic analysis of control or miR-26a inhibitor-transfected HaCaT cells with and without UVB irradiation. (I) Real-time PCR analysis of BAK, BAX, and BCL2 in control or miR-26a knockdown HaCaT cells with and without UVB irradiation.

miR-26a inhibitor was used to decrease its expression in HaCaT cells (Fig. 1G). After UVB irradiation for $48 \mathrm{~h}$, the apoptotic proportion of the miR-26a-depleted cells was half that in control group (Fig. 1H). Correspondingly, expression of BAK and BAX was decreased, while the expression of BCL2 was increased by miR-26a depletion upon UVB irradiation (Fig. 1I). Taken together, these results demonstrate that miR-26a positively regulates UVB-elicited apoptosis, suggesting that miR-26a is important for the elimination of UVB-damaged cells.

\section{EZH2 is a direct target of miR-26a in UVB-induced apoptosis}

Numerous studies have shown that miR-26a inhibits cell growth and tumorigenesis by targeting EZH2 [19, 21-24]. To test whether EZH2 was a direct target of miR-26a in UVBinduced apoptosis, we constructed wild type (WT) and mutant 3'-UTRs of human EZH2 (Fig. $2 \mathrm{~A}$ ) into miRNA reporters, and these plasmids were co-transfected with miR-26a mimic for 
A

EZH2 3'UTR

WT 5'-CTtTGaATAAagaATACTTGAA-3'

|| ||||||||

miR-26a 3'-UCGgaUAGgACCUAAUGAACUU-5'

Mutant 5'-CTTTGAATAAAGAAATGAAGCC-3'

C

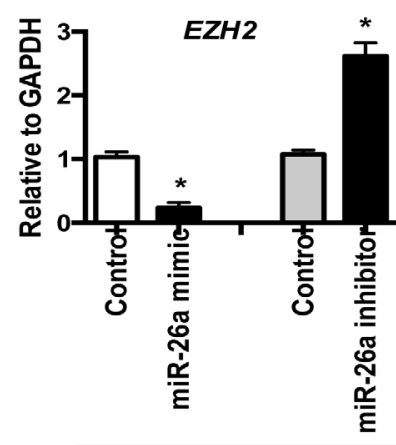

EZH2

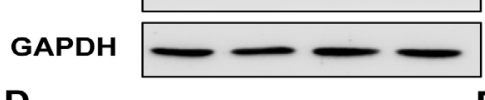

D

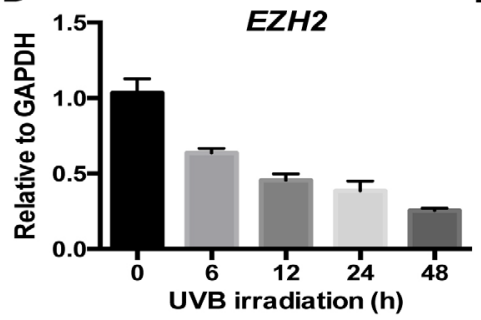

E

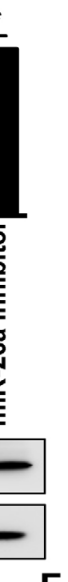

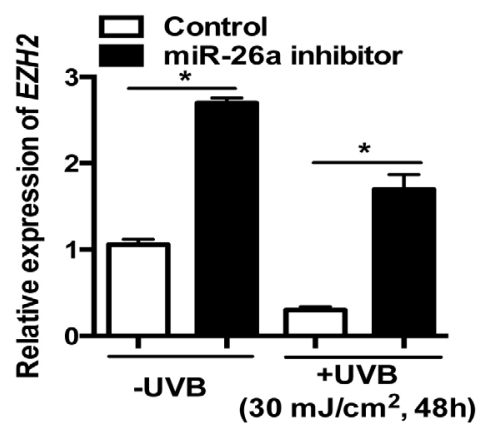
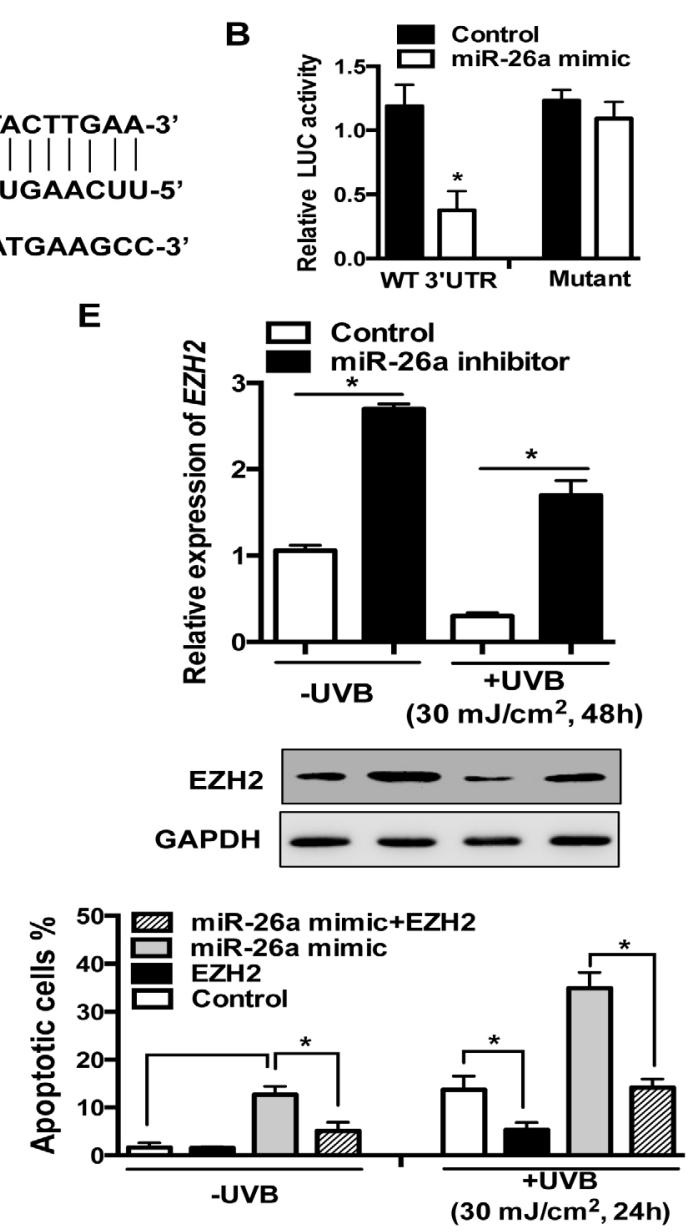

Fig. 2. EZH2 is a direct target of miR-26a in UVB-induced apoptosis(A) Schematic of $3^{\prime}$-UTR of EZH2 (WT) pairing with miR-26a. The mutant $3^{\prime}$-UTR of EZH2 is shown below. (B) pRL-TK and control or miR-26a mimic were co-transfected in HaCaT cells with miRNA luciferase reporters containing a WT or mutant $3^{\prime}$ UTR of EZH2. After $48 \mathrm{~h}$ of transfection, dual luciferase assays were conducted. (C) Real-time PCR analysis of the effects of miR-26a overexpression (miR-26a mimic) or knockdown (miR-26a inhibitor) on EZH2 expression. (D) Control or miR-26a inhibitor-transfected cells with and without UVB stimulation were subjected to apoptotic analysis. (E) Real-time PCR detection of EZH2 expression after 0, 6, 12, 24, and 48 h UVB irradiation. (F) The miR-26a mimic was transfected into HaCaT cells for $24 \mathrm{~h}$, alone or with either pcDNA3EZH2 or a control vector (pcDNA3), then exposed to UVB irradiation for $24 \mathrm{~h}$ before apoptotic analysis was conducted.

luciferase assays. As expected, miR-26a remarkably decreased WT but not mutant EZH2 3'UTR activity (Fig. 2B). Next, miR-26a mimic or inhibitor was transfected into HaCaT cells for determination of the EZH2 mRNA and protein expression. As shown in Fig. 2C, for both mRNA and protein, the miR-26a mimic decreased the expression of EZH2, while the miR-26a inhibitor upregulated EZH2 expression.

To examine the correlation between EZH2 and UVB irradiation, EZH2 expression was observed during a time course of UVB treatment. In contrast to miR-26a (Fig. 1B), EZH2 expression was decreased by UVB irradiation in a time-dependent manner (Fig. 2D). When miR-26a expression was knocked down, EZH2 mRNA and proteins levels were significantly upregulated with or without UVB treatment (Fig. 2E). Considering the negative correlation between miR-26a and EZH2, miR-26a depletion may release its inhibitory effect on EZH2, which may possess an opposing role in UVB-induced apoptosis. As shown in Fig. 2F, EZH2 


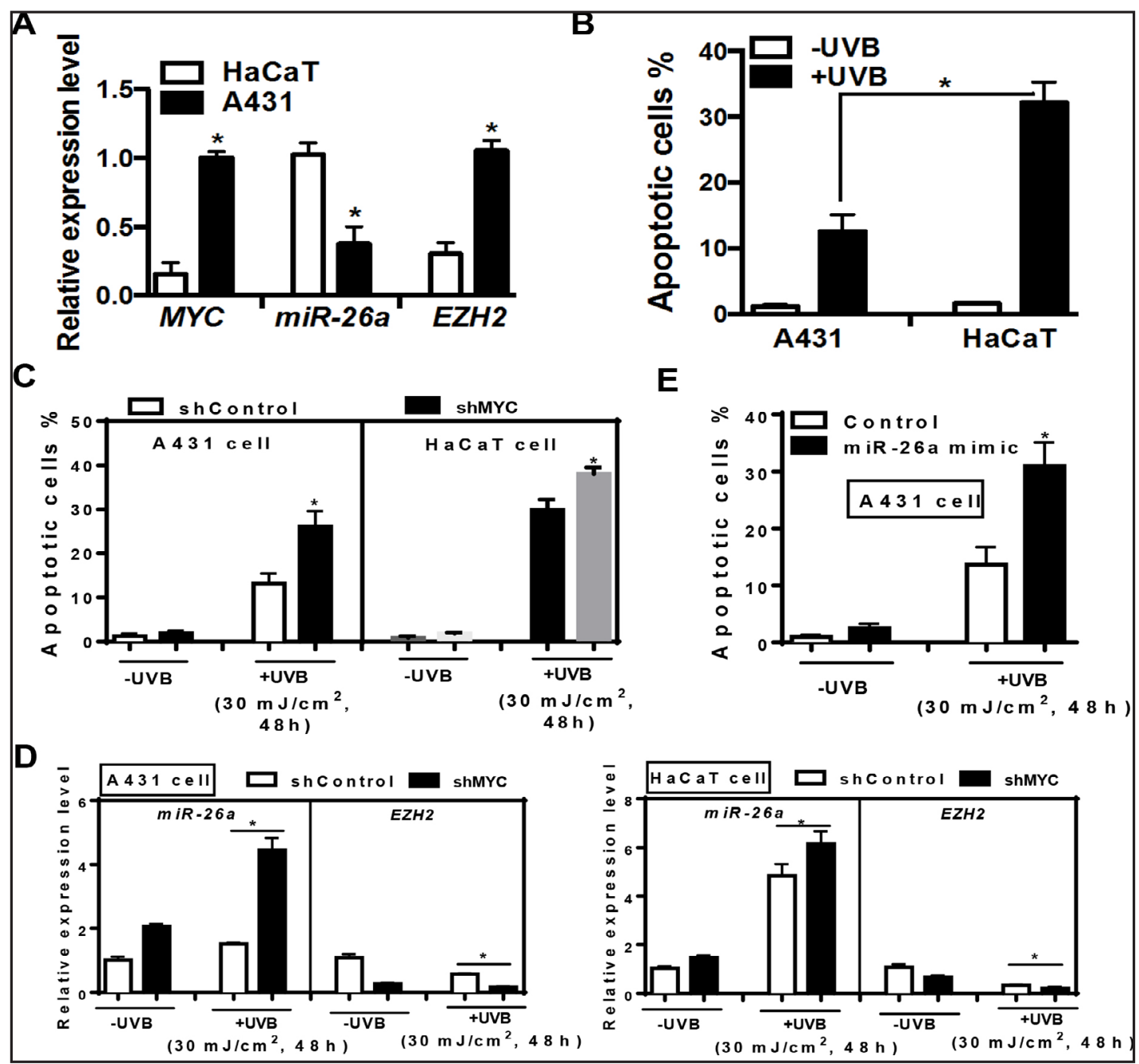

Fig. 3. Induction of miR-26a by UVB depends on MYC expression(A) Real-time PCR analysis of MYC, EZH2, and miR-26a expression in HaCaT and A431 cells. (B) Apoptotic analysis of A431 and HaCaT cells with and without 48 h of UVB irradiation. (C) MYC was depleted in A431 cells using a MYC-specific shRNA (shMYC), and apoptotic analysis was performed with and without $48 \mathrm{~h}$ of UVB irradiation. (D) Control (shControl)- or shMYC-transfected A431 cells were exposed to UVB irradiation for $48 \mathrm{~h}$, followed by real-time PCR analysis of miR-26a and EZH2 expression. (E) Control or miR-26a-overexpressed A431 cells were subjected to apoptotic analysis with and without $48 \mathrm{~h}$ of UVB irradiation.

overexpression antagonized both UVB- and miR-26a overexpression-induced apoptosis in $\mathrm{HaCaT}$ cells. This suggests that EZH2 functions downstream of miR-26a in the regulation of UVB-induced apoptosis.

Induction of miR-26a by UVB depends on MYC repression

It has been proposed that MYC-driven miRNA alterations contribute to tumorigenesis [25]. We compared the gene expression patterns of HaCaT cells, which are immortalized keratinocytes, with those of A431 epidermoid carcinoma cells. Interestingly, MYC and EZH2 were highly expressed in A431 cells, while miR-26a was highly expressed in HaCaT cells (Fig. 3A). We also examined the effects of UVB-induced apoptosis in A431 and HaCaT cells, and observed a much lower percentage of apoptosis in A431 cells (Fig. 3B). This indicates that MYC expression might be negatively associated with UVB/miR-26a-induced apoptosis. To test this notion, we depleted MYC in A431 and HaCaT cells, using a specific short hairpin 


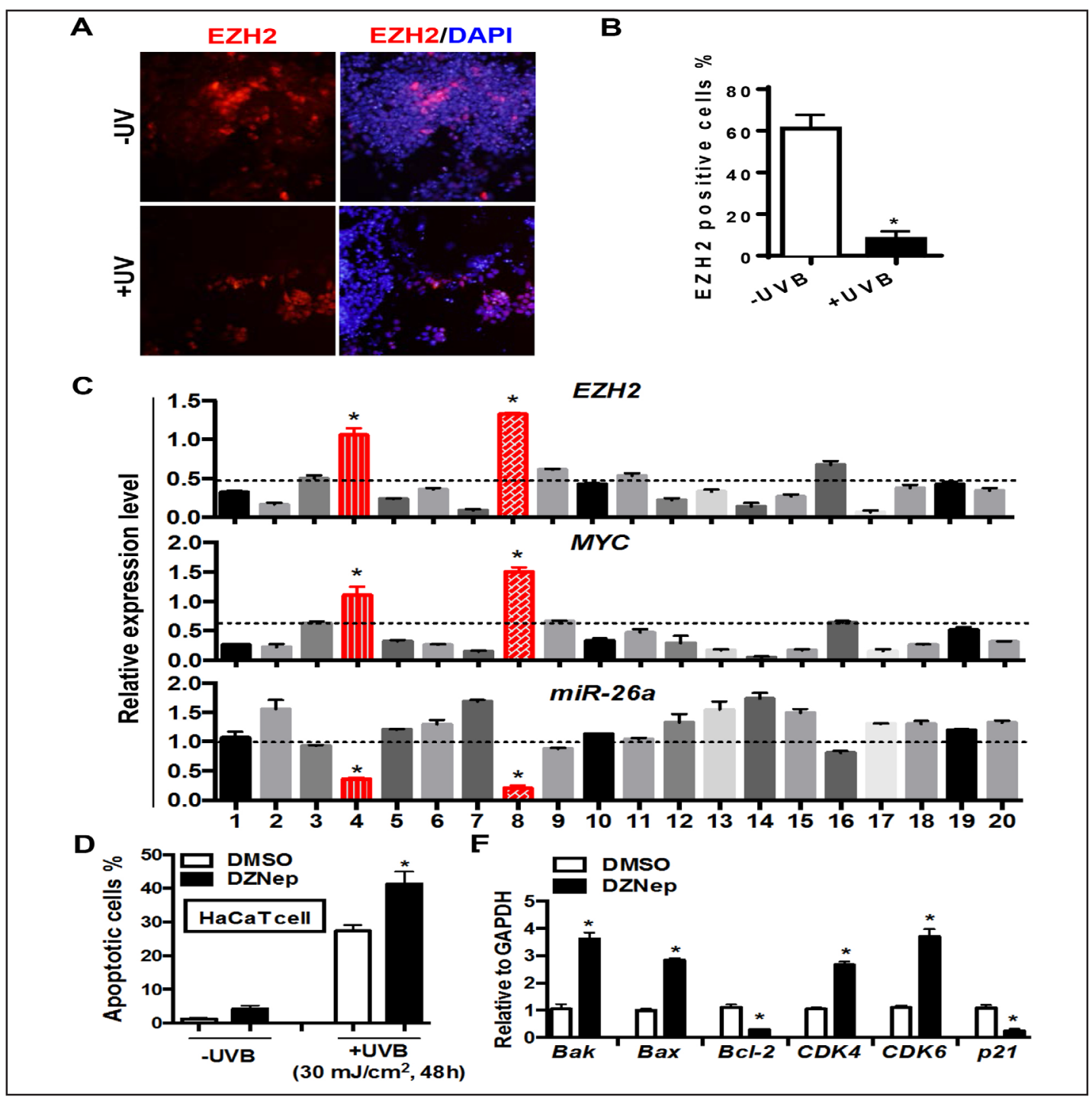

Fig. 4. An EZH2 inhibitor enhances UVB-induced apoptosis(A) HaCaT cells treated with UVB for 48 h were subjected to immunostaining with an anti-EZH2 antibody (red), and counterstained with DAPI (blue). Cells without UVB stimulation served as a control. (B) The proportion of EZH2-positive cells was calculated as the ratio between EZH2- and DAPI-positive cells. (C) Single cell PCR analysis of EZH2, MYC, and miR-26a expression in 20 UVB-irradiated HaCaT cells. The red bars indicate cells with high EZH2 and MYC expression and low miR-26a expression. (D) Control or UVB-stimulated HaCaT cells were treated with DMSO or DZNep $(1 \mu \mathrm{M})$ for $48 \mathrm{~h}$ before apoptotic analysis. (E) DMSO- or DZNep $(1 \mu \mathrm{M})$-treated cells irradiated with UVB were collected for real-time PCR analysis of BAK, BAX, BCL2, CDK4, CDK6, and p21 expression.

RNA (shRNA), and found that MYC depletion remarkably enhanced UVB-induced apoptosis (Fig. 3C). Next, the relationship between MYC and the miR-26a/EZH2 regulatory axis was investigated. Interestingly, depletion of MYC expression in A431 and HaCaT cells promoted miR-26a expression and suppressed EZH2 expression, with or without UVB irradiation (Fig. 3D). Furthermore, to determine if the lower miR-26a level in A431 cells is causally involved in their decreased sensitivity upon UVB irradiation, we overexpressed miR-26a in A431 cells, and observed increased apoptosis in response to UVB treatment (Fig. 3E). Taken together, these data demonstrate that miR-26a-induced changes in EZH2 expression depend on MYC expression in UVB-irradiated cells. 


\section{Cellular Physiology Cell Physiol Biochem 2017;43:1188-1197

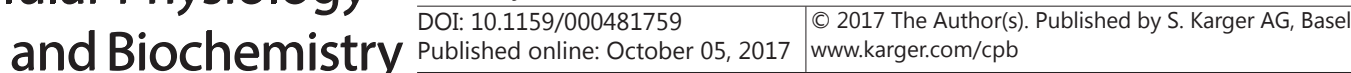 \\ Zhang et al.: Mir-26a Mediates Apoptosis in UVB Irradiated Cells}

\section{EZH2 inhibition enhances UVB-induced apoptosis}

To confirm the observed changes in EZH2 expression in response to UVB treatment, immunostaining with an anti-EZH2 antibody was conducted in HaCaT cells. In control cells without UVB irradiation, approximately $60 \%$ of cells were EZH2-positive. However, after UVB irradiation for $48 \mathrm{~h}$, the proportion of EZH2-positive cells was significantly decreased, to approximately $9 \%$ (Fig. 4A and 4B).

Our results suggest that co-expression of EZH2 and MYC may allow cells to escape apoptosis after UVB irradiation. To examine this possibility, we used single cell PCR, which has been widely used to study the heterogeneity of cancer cells and other populations [26]. UVB-irradiated HaCaT cells were trypsinized into single cells and 20 cells were randomly selected for single cell reverse transcription using the Smart-seq2 method [20]. Two cells showed relatively higher expression of EZH2, and these cells had higher expression of MYC and lower expression of miR-26a (Fig. 4C), indicating that upregulation of miR-26a upon UVB irradiation might be blocked by MYC. We next asked whether inhibition of EZH2 might be helpful to eliminate UVB-damaged cells. We treated HaCaT cells with $1 \mu \mathrm{M}$ DZNep, an inhibitor of EZH2 histone methyltransferase activity, and found that DZNep significantly enhanced the ability of UVB to induce cell apoptosis (Fig. 4D). Moreover, DZNep treatment promoted the expression of BAK, BAX, CDK4, and CDK6, while inhibiting the expression of BCL2 and p21 (Fig. 4E). Collectively, these data suggest that inhibition of EZH2 activity in UVB-treated cells could help to eliminate UVB-damaged cells with higher expression of MYC. The expression of cell cycle-related genes was also affected by DZNep, indicating that the $\mathrm{MYC} / \mathrm{miR}-26 \mathrm{a} / \mathrm{EZH} 2$ regulatory axis might also be involved in cell cycle regulation.

\section{Discussion}

The regulation of gene expression by miRNAs plays important roles in skin development and carcinogenesis [27]. It has been proposed that miR-26a inhibits proliferation and migration of HaCaT keratinocytes by targeting PTEN [19]. In this study, for the first time, we demonstrate that miR-26a mediates UVB-induced apoptosis, targeting the histone methyltransferase EZH2 in a MYC-dependent manner.

Our results suggest a role for miR-26a, which is upregulated by UVB irradiation, in the elimination of UVB-damaged cells, avoiding proliferation and, potentially, the development of skin cancer. This suggests that miR-26a may act as a tumor suppressor in UVB-triggered skin carcinogenesis.

Reports suggest that miR-26a suppresses the proliferation of keratinocytes, yet promotes the proliferation of vascular smooth muscle cells and ovarian cancer cells, demonstrating the complexity of miR-26a, which functions in different biological processes through different targets $[19,28-30]$. In the present study, we identified EZH2 as a direct target of miR-26a in UVB-induced apoptosis, and found that it antagonized miR-26a-induced apoptosis upon UVB irradiation (Fig. 2). We postulate that suppression of EZH2 by miR-26a is necessary for the elimination of UVB-damaged cells.

By comparing gene expression patterns in HaCaT keratinocytes and A431 epidermoid carcinoma cells, we found that MYC and EZH2 had higher expression in A431 carcinoma cells (Fig. 3A). It has been proposed that MYC could repress miR-26a expression to stimulate EZH2 transcription [22]. Our findings are consistent with this, and suggest that MYC acts upstream of miR-26a/EZH2, and that high MYC expression may protect A431 cells from UVB-induced apoptosis.

Importantly, blocking EZH2 activity with DZNep enhanced UVB-elicited apoptosis (Fig. 4), demonstrating that inhibition of EZH2 could bypass the failure to eliminate these UVBirradiated cells. With high MYC expression, the efficiency of miR-26a to target EZH2 and induce apoptosis in response to UVB radiation was restricted, allowing these damaged cells to evade apoptosis. The perturbation of apoptotic responses to UVB has important clinical implications for skin carcinogenesis.

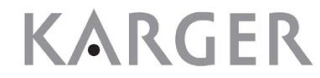




\section{Cellular Physiology Cell Physiol Biochem 2017;43:1188-1197

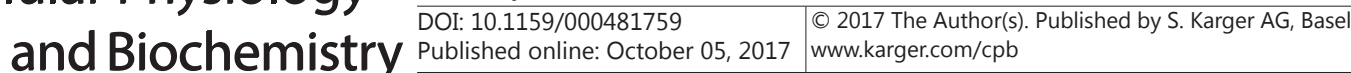 \\ Zhang et al.: Mir-26a Mediates Apoptosis in UVB Irradiated Cells}

In summary, we have demonstrated that miR-26a can promote UVB-induced apoptosis by targeting the histone methyltransferase EZH2, provided that MYC expression is repressed. Notably, inhibition of EZH2 leads to enhanced apoptosis upon UVB irradiation, suggesting that EZH2 might be a potential target for skin cancer prevention and therapy.

\section{Acknowledgements}

This study was supported by the National Natural Science Foundation of China (No. 81301380, 81573072).

\section{Disclosure Statement}

The authors declare that they have no competing interests

\section{References}

1 Marrot L, Meunier JR: Skin DNA photodamage and its biological consequences. J Am Acad Dermatol 2008;58:S139-S148.

2 Timares L, Katiyar SK, Elmets CA: DNA damage, apoptosis and langerhans cells--Activators of UV-induced immune tolerance. Photochem Photobiol 2008;84:422-436.

3 Lomas A, Leonardi-Bee J, Bath-Hextall F: A systematic review of worldwide incidence of nonmelanoma skin cancer. Br J Dermatol 2012;166:1069-1080.

4 Miralles F, Parra M, Caelles C, Nagamine Y, Felez J, Munoz-Canoves P: UV irradiation induces the murine urokinase-type plasminogen activator gene via the c-Jun $\mathrm{N}$-terminal kinase signaling pathway: requirement of an AP1 enhancer element. Mol Cell Biol 1998;18:4537-4547.

5 Gordon R: Skin cancer: an overview of epidemiology and risk factors. Semin Oncol Nurs 2013;29:160-169.

-6 Herzinger T, Funk JO, Hillmer K, Eick D, Wolf DA, Kind P: Ultraviolet B irradiation-induced G2 cell cycle arrest in human keratinocytes by inhibitory phosphorylation of the cdc2 cell cycle kinase. Oncogene 1995;11:2151-2156.

7 Ziegler A, Jonason AS, Leffell DJ, Simon JA, Sharma HW, Kimmelman J, Remington L, Jacks T, Brash DE: Sunburn and p53 in the onset of skin cancer. Nature 1994;372:773-776.

8 Jackson S, Harwood C, Thomas M, Banks L, Storey A: Role of Bak in UV-induced apoptosis in skin cancer and abrogation by HPV E6 proteins. Genes Dev 2000;14:3065-3073.

-9 Guo L, Huang ZX, Chen XW, Deng QK, Yan W, Zhou MJ, Ou CS, Ding ZH: Differential expression profiles of microRNAs in NIH3T3 cells in response to UVB irradiation. Photochem Photobiol 2009;85:765-773.

10 Pothof J, Verkaik NS, Hoeijmakers JH, van Gent DC: MicroRNA responses and stress granule formation modulate the DNA damage response. Cell Cycle 2009;8:3462-3468.

-11 Pothof J, Verkaik NS, van IJcken W, Wiemer EA, Ta VT, van der Horst GT, Jaspers NG, van Gent DC, Hoeijmakers JH, Persengiev SP: MicroRNA-mediated gene silencing modulates the UV-induced DNAdamage response. EMBO J 2009;28:2090-2099.

12 Li W, Zhou BR, Hua LJ, Guo Z, Luo D: Differential miRNA profile on photoaged primary human fibroblasts irradiated with ultraviolet A. Tumour Biol 2013;34:3491-3500.

13 Guo Z, Zhou B, Liu W, Xu Y, Wu D, Yin Z, Permatasari F, Luo D: MiR-23a regulates DNA damage repair and apoptosis in UVB-irradiated HaCaT cells. J Dermatol Sci 2013;69:68-76.

14 Li W, Di W, Hua L, Zhou B, Guo Z, Luo D: UVB suppresses PTEN expression by upregulating miR-141 in HaCaT cells. J Biomed Res 2011;25:135-140.

15 Li W, Wu YF, Xu RH, Lu H, Hu C, Qian H: miR-1246 releases RTKN2-dependent resistance to UVB-induced apoptosis in HaCaT cells. Mol Cell Biochem 2014;394:299-306.

16 Lin Y, Chen H, Hu Z, Mao Y, Xu X, Zhu Y, Xu X, Wu J, Li S, Mao Q, Zheng X, Xie L: miR-26a inhibits proliferation and motility in bladder cancer by targeting HMGA1. FEBS Lett 2013;587:2467-2473. 


\section{Cellular Physiology Cell Physiol Biochem 2017;43:1188-1197 \begin{tabular}{l|l|l} 
DOI: 10.1159/000481759 & $\begin{array}{l}\text { O 2017 The Author(s). Published by S. Karger AG, Basel } \\
\text { www.karger.com/cpb }\end{array}$ \\
\hline
\end{tabular}

-17 Liu B, Wu X, Liu B, Wang C, Liu Y, Zhou Q, Xu K: MiR-26a enhances metastasis potential of lung cancer cells via AKT pathway by targeting PTEN. Biochim Biophys Acta 2012;1822:1692-1704.

18 Sun TY, Xie HJ, He H, Li Z, Kong LF: miR-26a inhibits the proliferation of ovarian cancer cells via regulating CDC6 expression. Am J Transl Res 2016;8:1037-1046.

19 Yu N, Yang Y, Li X, Zhang M, Huang J, Wang X, Long X: MiR-26a inhibits proliferation and migration of HaCaT keratinocytes through regulating PTEN expression. Gene 2016;594:117-124.

-20 Picelli S, Faridani OR, Bjorklund AK, Winberg G, Sagasser S, Sandberg R: Full-length RNA-seq from single cells using Smart-seq2. Nat Protoc 2014;9:171-181.

-21 Lu J, He ML, Wang L, Chen Y, Liu X, Dong Q, Chen YC, Peng Y, Yao KT, Kung HF, Li XP: MiR-26a inhibits cell growth and tumorigenesis of nasopharyngeal carcinoma through repression of EZH2. Cancer Res 2011;71:225-233.

22 Sander S, Bullinger L, Klapproth K, Fiedler K, Kestler HA, Barth TF, Moller P, Stilgenbauer S, Pollack JR, Wirth T: MYC stimulates EZH2 expression by repression of its negative regulator miR-26a. Blood 2008;112:4202-4212.

23 Zhuang C, Wang P, Huang D, Xu L, Wang X, Wang L, Hu L: A double-negative feedback loop between EZH2 and miR-26a regulates tumor cell growth in hepatocellular carcinoma. Int J Oncol 2016;48:1195-1204.

24 Chen J, Xu Y, Tao L, Pan Y, Zhang K, Wang R, Chen LB, Chu X: MiRNA-26a Contributes to the Acquisition of Malignant Behaviors of Doctaxel-Resistant Lung Adenocarcinoma Cells through Targeting EZH2. Cell Physiol Biochem 2017;41:583-597.

25 Chang TC, Yu D, Lee YS, Wentzel EA, Arking DE, West KM, Dang CV, Thomas-Tikhonenko A, Mendell JT: Widespread microRNA repression by Myc contributes to tumorigenesis. Nat Genet 2008;40:43-50.

-26 Dalerba P, Kalisky T, Sahoo D, Rajendran PS, Rothenberg ME, Leyrat AA, Sim S, Okamoto J, Johnston DM, Qian D, Zabala M, Bueno J, Neff NF, Wang J, Shelton AA, Visser B, Hisamori S, Shimono Y, van de Wetering M, Clevers H, Clarke MF, Quake SR: Single-cell dissection of transcriptional heterogeneity in human colon tumors. Nat Biotechnol 2011;29:1120-1127.

27 Sand M, Gambichler T, Skrygan M, Sand D, Scola N, Altmeyer P, Bechara FG: Expression levels of the microRNA processing enzymes Drosha and dicer in epithelial skin cancer. Cancer Invest 2010;28:649-653.

28 Deng J, He M, Chen L, Chen C, Zheng J, Cai Z: The loss of miR-26a-mediated post-transcriptional regulation of cyclin E2 in pancreatic cancer cell proliferation and decreased patient survival. PLoS One 2013;8:e76450.

29 Zhou J, Ju W, Wang D, Wu L, Zhu X, Guo Z, He X: Down-regulation of microRNA-26a promotes mouse hepatocyte proliferation during liver regeneration. PLoS One 2012;7:e33577.

30 Zuo K, Zhi K, Zhang X, Lu C, Wang S, Li M, He B: A dysregulated microRNA-26a/EphA2 axis impairs endothelial progenitor cell function via the p38 MAPK/VEGF pathway. Cell Physiol Biochem 2015;35:477488. 